\title{
A study of a possible cancer cure by correcting the main cancer-related psychoneuroendocrine alterations responsible for tumor-related immunosuppression
}

\author{
Paolo Lissoni ${ }^{*}$, Giusy Messina ${ }^{2}$, Franco Rovelli ${ }^{1}$, Arianna Lissoni ${ }^{1}$, Luca Fumagalli ${ }^{3}$, Fernando Brivio ${ }^{4}$, Giorgio Porro ${ }^{1}$ and Giuseppe Di \\ Fede $^{1}$ \\ ${ }^{1}$ Institute of Biological Medicine, Milan, Italy \\ ${ }^{2}$ International Institute of PNEI, Italy \\ ${ }^{3}$ Surgical Division, Manzoni Hospital, Lecco, Italy \\ ${ }^{4}$ Surgical Division, Cinisello Hospital, Cinisello Balsamo, Milan, Italy
}

\begin{abstract}
It is known since more than 50 years that the pineal gland plays a fundamental anticancer physiological role by releasing hormones capable of counteract cancer growth by either inhibiting cancer cell proliferation, or stimulating the antitumor immunity, as well as that cancer progression is constantly associated with a progressive decline in the pineal endocrine function. Despite the well demonstrated antitumor activity of the pineal gland and the complete absence of biological toxicity and the low cost of the antitumor pineal molecules, surprisingly very few clinical studies have been carried out up to now with pineal hormones in the treatment of human neoplasms, at least in patients, for whom no other conventional anticancer therapy may be available. The present study was carried out to evaluate the therapeutic effects of a pineal endocrine substitution therapy with high-dose melatonin (MLT) alone ( $\mathrm{n}=68$ ), MLT plus 5-methoxytryptamine (5-MTT) ( $\mathrm{n}=72$ ) or MLT plus 5-MTT plus pinealine (PNL) $(\mathrm{n}=28)$ in 168 untreatable metastatic solid tumor patients with life expectancy less than 1 year. A survival longer than 1 year was achieved in 72/168 (43\%), and both objective tumor regression rate and 1-year survival observed in patients treated with MLT plus other pineal hormones were higher than in patiente treated by MLT alone, without, however, statistically significant differences. On the contrary, the percentage of disease control (DC) obtained by MLT plus other pineal molecules was significanly higher than that achieved by MLT alone. The clinical status improved in most patients. Finally, lymphocyte count and lymphocyte-to-monocyte ratio enhanced in patients with DC, whereas a further decline occurred in patients with PD. This preliminary study show that a pineal endocrine therapy may improve the prognosis of metastatic cancer patients, including those for whom no other standard anticancer therapy is available, and that the control of the neoplastic disease may be further enhanced by associating to MLT other less investigated antitumor pineal hormones.
\end{abstract}

\section{Introduction}

After of a long time of conflicting results, today their is no doubt about the possibility to treat the human neoplasms by acting on the immune system [1]. The control of cancer growth by stimulating host anticancer immunity is now a real clinical approach on the basis of three main evidence, consisting of the immunosuppressive affect of macrophage-mediated chronic inflammatory response [2], the generation of regulatory $\mathrm{T}$ lymphocytes ( $\mathrm{T}$ reg) able to suppress the anticancer immunity [3], and the existence of immune inhibitory checkpoints, mainly consisting of CTLA-4 [4], PD-1 and its ligands PD-L1 and PD-L2 [5], which are expressed by T lymphocytes and tumor cells themselves, and whose activation may suppress both antigen- specific and non-specific anticancer immunity [6]. The in vivo activation of an effective anticancer immunity may be obtained through the administration of antitumor cytokines, such as IL-2 [7] and IL-12 [8], or that of anti-inhibitory checkpoint monoclonal antibodies [4,5]. However, more recent investigtions have shown that the in vivo immune responses, including the anticancer immunity, is physiologically under a psychoneuroendocrine control $[9,10]$, which is the neurochemical expression of the psychospiritual life [11]. In more detail, the anticancer immunity has appeared to be inhbited by the brain opioid system, mainly through mu-opioid receptor [12], and to be stimulated by the pineal gland through its main indole hormone melatonin (MLT) [13] and other less investigated indole and beta-carboline molecules [14,15]. Moreover, it is known since many years that cancer-progression is constantly associated with a progressive decline in the pineal endocrine function in both animals and humans [16], with a following progressive decline in the nocturnal production of MLT. Because of the stimulatory effect of MLT on the anticancer immunity, with stimulatory effects of T-helper lymphocytes (TH) and inhibitory ones on macrophagemediatd immunosuppression [13], the main cancer-related immune alterations, including low levels of IL-2 and high concentrations of IL-6 [17], could depend at least in part on the progressive cancer-related pineal hypofunction and low MLT blood levels. In fact, preliminary clinical studies have shown that the chronic administration of mild pharmacological doses of MLT, consisting of about $20 \mathrm{mg}$, once / daily during the dark period, may improve the immune status and prolong the survival time in metastatic cancer patients with life expectancy less than 6 months, and for whom no other standard anticancer treatment

${ }^{*}$ Correspondence to: Paolo Lissoni, Institute of Biological Medicine, Milan, Italy, E-mail: paolo.lissoni@gmx.com

Key words: beta-carbolines, cancer cure, melatonin, 5-methoxytryptamine, pineal gland, pinealine

Received: March 04, 2019; Accepted: April 25, 2019; Published: April 29, 2019 
Lissoni P (2019) A study of a possible cancer cure by correcting the main cancer-related psychoneuroendocrine alterations responsible for tumor-related immunosuppression

was available $[18,19]$. Moreover, the association of MLT has been proven to enhance the antitumor efficacy of IL-2 cancer immunotherapy [20]. However, it has to be remarked that MLT is not the only hormone produced by the pineal gland, since at least two other pineal molecules have appeared to play anticancer activity, the 5-methoxytryptamine (5-MTT) and the beta-carboline 6-methoxy-1,2,3,4-tetrahydro-beta carboline, also called pinealine (PNL) $[14,15]$. Histological damages of the pineal gland have been observed in patients died from cancer [21]. Then, it is probable that cancer progression-related pineal deficiency does not involve the only MLT secretion, but that of the overall pineal hormones. Therefore, an eventual pineal substitutive endocrine therapy would include at least the administration of MLT, 5-MTT and PNL. In addition, the anticancer effects of MLT has been recently proven to be a dose-dependent phenomenon in humans [19]. On this basis, a clinical study was plannedto evaluate the therapeutic anticancer effects of MLT alone, MLT plus 5-MTT, and MLT plus 5-MTT and PNL in metastatic cancer patients, for whom no other conventional anticancer therapy was available.

\section{Materials and methods}

The study was performed to compare the results achieved during different clinical investigations in three different group o untreatable metastatic solid tumor cancer patients, who had received high-dose MLT alone (group A; $\mathrm{n}=68$ ), high-dose MLT plus 5-MTT (group B; $\mathrm{n}=72$ ), and high-dose MLT plus 5-MTT plus PNL (group C; $\mathrm{n}=28$ ), for a total of 168 patients. MLT was given at very-pharmacologically doses because of its demonstrated dose-depedent anticancer potency in humans [19]. On the contrary, 5-MTT and PNL were given at doses corresponding about to their whole daily production with substitute intention in an attempt to correct a probable their deficiency with cancer progression, as shown in experimental conditions [16,21]. The experimental protocol was explained to the patients and their consent was obtained. Eligibility criteria were, as follows: histologically proven metastatic solid tumor, measurable lesions, no availability of other standard anticancer therapy, including chemotherapy, endocrine therapy, anti-angiogenic treatments, target therapy and immuotherapy, because of lack of response to previous treatments or poor clinical conditions unable to substain specific anticancer therapies, no abdominal occlusion and life expectancy less than 1 year or less than 6 months in some cases. All treatments were given orally and once/daily in relation to the different phases of the photoperiod. MLT was given at $100 \mathrm{mg} /$ day in the dark, about 30 minutes before sleeping, 5-MTT was given at $5 \mathrm{mg} /$ day in the period of light at about 1 P.M, and finally PNL was given at $1 \mathrm{mg} /$ day at about 8 P.M. or about 3 hours before sleeping. The treatment was continued without interruption until disease progression, by repeating the main radiological examinations every 3 months for the first year, including CT scan, NMR and PET, then every 6 months. In addition as palliative therapy because of their potential anti-inflammatory and antalgic effects [22-24], the supportive treatment consisted of Aloe arborescens/Myrrh mixture (60:40) at 10 $\mathrm{ml}$ twice/day and Magnolia cortex with honokiol concentration more than $50 \%$ at $500 \mathrm{mg}$ twice/day. The clinical results were evaluated according to WHO criteria at 3-month intervals. Moreover, because of its recently demonstrated capacity of reflecting the anticancer immune status of patients, from a laboratoristic point of view, patients were monitored by evaluating lymphocyte-to-monocyte ratio (LMR) as usefull inexpensive biomarker provided by prognostic significance [25]. Normal values obtained in our laboratory (95\% confidence limits) were more than $1,500 / \mathrm{mm} 3$ for lymphocytes and values grearter than 2.1 for the LMR. Data were reported as mean +/- SE, and statistically analyzed by the chi-square test and the Student's te test, as appropriate.

\section{Results}

The clinical characteristics of the three groups of patients are reported in Table 1. As shown, the three groups of patients were well balanced for the main prognostic clinical variables, including age, disease extension, tumor histotype, metastatic sites and clinical status. The clinical results achieved in the different groups of patients are reported in Table 2. According to WHO criteria, a complete response (CR) was obtained in 4/168 (2\%) patients (group A:1 (biliary tract cancer); group B: 2 (lung adenocarcinoma: 2); group C: 1 (gastric cancer)). A partial response occurred in other 12/168 (7\%) patients (group A: 3 (biliary tract cancer:1; colon cancer:1; pancreatic cancer:1); group B: 6 (colorectal carcinoma: 2; epidermoid lung cancer:1; pancreatic cancer: 1; bladder cancer:1; biliary tract cancer:1); group C: 3 (melanoma:1; triple negative breast cancer:1; colorectal cancer:1). Therefore, an objective tumor regression (CR + PR) was obtained in $16 / 168$ (9\%) patients. Moreover, tumor regression rate obtained in the groups B and C, treated respectively by MLT plus 5-MTT and MLT plus 5-MTT plus PNL, was higher with respect to that found in the group A treated with the only MLT, without, however, statistically significant differences (group A: 4/68 (6\%); group B: 8/72 (11\%); group C: $4 / 28(14 \%)$ ). A stable disease (SD) occurred in $90 / 168$ (54\%) patients (group A: 31/68 (46\%); group B: 41/72 (57\%); group C: 18/28 (64\%)). The, disease control (DC), including CR, PR and SD, was obtained in $106 / 168(63 \%)$, whereas the ramaining $62 / 168(37 \%)$ patients had a progressive disease (PD). Moreover, the percentage of DC achieved in patients concomitantly treated by MLT plus 5-MTT was statistically significantly higher than that found in patients, who received MLT alone $(49 / 72(68 \%)$ vs $35 / 68$ (51\%), P 0.05). The higher percentage of DC was observed in the group C treated by MLT plus 5-MTT plus PNL (JHK22/29 (78\%)), even though the difference was not statistically significant with respect to the results found in the group B of MLT plus 5-MTT because of the low number of group C patients. Finally, as far as the survival time, after a follow up of at least 1 year, a survival longer than 1 year was obtained in 72/168 (43\%). The percentage of 1-year survival was greater in groups $\mathrm{B}$ and $\mathrm{C}$ than in the group $\mathrm{A}$, even thoug the difference did not reach the statistical significance. Almost all patients of the three groups referred an improvement in mood, sleep disorders, asthenia, and anorexia. The most evident improvement was seen in the group of patients also treated by PNL, particularly in the self-consciousness state. A low simptomatology of extra-sensorial perceptions was referred by $2 / 28(8 \%)$ patients treated with PNL, without, however, any concomitant anxiety. Finally, as far as the immunobiological profile is concerned, as shown in Table 3, irrespectively of the type of therapy, both lymphocyte count and LMR pretreatment mean values were lower in patients who had a PD than in those with DC, without, however, statistically significant differences. On the contrary, both lymphocyte meam mumber and and LMR mean values observed after three months of therapy at the same time of the first radiological evaluation were significantly higher in patients who achieved a DC than in those, who had a PD within the same group of therapy.

\section{Discussion}

This preliminary clinical study, carried out in metastatic cancer patients with life expectancy less than 1 year because of no availability of other standard antitumor therapies, would show a progressive increase in the antitumor efficacy of a pineal endocrine cancer therapy by a progressive substitution treatment with pineal indole other than MLT, such as 5-MTT, and pineal antitumor beta-carbolines, such as PNL, in an attempt to counteract a probable cancer progression- 
Lissoni P (2019) A study of a possible cancer cure by correcting the main cancer-related psychoneuroendocrine alterations responsible for tumor-related immunosuppression

Table 1. Clinical characteristics of metastatic cancer patients treated with melatonin (MLT) alone (group A), MLT plus 5-methoxytryptamine (5-MTT) (group B), and MLT plus 5-MTT plus pinealine (PNL) (group C)

\begin{tabular}{|c|c|c|c|c|}
\hline Characteristics & $n(n=168)$ & $\operatorname{MLT}(n=68)$ & $\begin{array}{c}\text { MLT + 5-MTT } \\
(\mathrm{n}=72)\end{array}$ & $\begin{array}{r}\text { MLT + 5-MTT } \\
+ \text { PNL }(n=28)\end{array}$ \\
\hline Median age (years) & & $68(42-85)$ & $65(34-92)$ & $67(44-86)$ \\
\hline $\operatorname{Sex}(\mathrm{M} / \mathrm{F})$ & & $38 / 30$ & $37 / 35$ & $13 / 15$ \\
\hline Median PS (Ecog) & & $1(0-3)$ & $1(0-3)$ & $1(0-3)$ \\
\hline \multicolumn{5}{|l|}{ Tumor histotype } \\
\hline - Lung cancer & 35 & 16 & 15 & 4 \\
\hline - Colorectal cancer & 25 & 9 & 12 & 4 \\
\hline - Pancreas cancer & 26 & 10 & 11 & 5 \\
\hline - Gastric cancer & 10 & 4 & 5 & 1 \\
\hline - Biliary tract cancer & 20 & 11 & 7 & 2 \\
\hline - Urinary tract tumors & 9 & 4 & 3 & 2 \\
\hline - Gynecologic tumors & 15 & 6 & 6 & 3 \\
\hline - Breast cancer & 10 & 2 & 4 & 4 \\
\hline - Triple negative & 6 & 0 & 2 & 4 \\
\hline - No triple negative & 4 & 2 & 2 & 0 \\
\hline - Soft tissue sarcoma & 10 & 4 & 5 & 1 \\
\hline - Melanoma & 8 & 2 & 4 & 2 \\
\hline \multicolumn{5}{|l|}{$\begin{array}{l}\text { Distant organ } \\
\text { metastas }\end{array}$} \\
\hline -Soft tissues & 15 & 5 & 8 & 2 \\
\hline -Bone & 11 & 6 & 4 & 1 \\
\hline -Lung & 36 & 13 & 15 & 8 \\
\hline -Liver & 43 & 18 & 17 & 8 \\
\hline -Liver plus lung & 21 & 9 & 8 & 4 \\
\hline -Peritoneum & 18 & 8 & 8 & 2 \\
\hline -Brain & 24 & 9 & 12 & 3 \\
\hline
\end{tabular}

Table 2. Clinical response (WHO criteria), and 1-year survival in untreatable metastatic solid tumor patients treated by melatonin (MLT) alone (group A), MLT plus 5-methoxytryptamine (5-MTT) (group B) or MLT plus 5-MTT plus pinealine (PNL)

\begin{tabular}{|c|c|c|c|c|c|c|c|c|}
\hline \multicolumn{2}{|l|}{ Patients } & \multicolumn{6}{|c|}{ Clinical response $^{*}$} & \multirow{2}{*}{$\begin{array}{c}\text { 1-year } \\
\text { survival }\end{array}$} \\
\hline & $\mathbf{n}$ & CR & PR & $\begin{array}{c}\text { CR + } \\
\text { PR }\end{array}$ & SD & DC & PD & \\
\hline $\begin{array}{c}\text { Group A (MLT) } \\
\text { Group B (MLT + 5MTT) } \\
\text { Group C (MLT+5- } \\
\text { MTT+PNL) }\end{array}$ & $\begin{array}{l}68 \\
72 \\
28\end{array}$ & $\begin{array}{l}1 \\
2 \\
1\end{array}$ & $\begin{array}{l}3 \\
6 \\
3\end{array}$ & $\begin{array}{l}4(6 \%) \\
8(11 \%) \\
4(14 \%)\end{array}$ & $\begin{array}{l}31 \\
41 \\
18\end{array}$ & $\begin{array}{l}35(51 \%) \\
49(68 \%)^{* * *} \\
22(78 \%)^{* * *}\end{array}$ & $\begin{array}{c}33 \\
23 \\
6\end{array}$ & $\begin{array}{l}26(38 \%) \\
3143 \%) \\
15(53 \%)\end{array}$ \\
\hline
\end{tabular}

${ }^{*} \mathrm{CR}$ : Complete response; PR: Partial response; SD: Stable disease; DC: Disease control; PD: Progressive disease.

${ }^{* *} \mathrm{P}<0.05$ vs group $\mathrm{A} ; \mathrm{P}<0.01$ vs group $\mathrm{A}$.

Table 3. Lymphocyte mean number and lymphocyte-to-monocyte ratio (LMR) before therapy and after 3 months of treatment in metastatic solid tumor patients treated with MLT, MLT + 5-MTT or MLT + 5-MTT plus PNL in relation to the clinical response, consisting of progressive disease (PD) or disease control (DC)

\begin{tabular}{|c|c|c|c|c|c|}
\hline \multirow{2}{*}{ Patients } & \multirow{2}{*}{$\mathbf{n}$} & \multicolumn{2}{|c|}{ Lymphocytes n/mm3 ( $\mathrm{X} \pm \mathrm{SE})$} & \multicolumn{2}{|c|}{ LMR ( \pm \pm SE) } \\
\hline & & Before & After & Before & After \\
\hline MLT & 68 & $1,346 \pm 188$ & $1,541 \pm 165$ & $2.3 \pm 0.3$ & $2.5 \pm 0.4$ \\
\hline -DC & 35 & $1,585 \pm 216$ & $1,788 \pm 141$ & $2.7 \pm 0.4$ & $3.3 \pm 0.3^{*}$ \\
\hline$-\mathrm{PD}$ & 33 & $1,208 \pm 209$ & $1,176 \pm 215$ & $2.1 \pm 0.5$ & $1.8 \pm 0.4$ \\
\hline MLT+5-MTT & 72 & $1,442 \pm 176$ & $1,544 \pm 161$ & $2.4 \pm 0.4$ & $2.8 \pm 0.3$ \\
\hline -DC & 49 & $1,639 \pm 164$ & $1,848 \pm 144$ & $2.8 \pm 0.5$ & $3.6 \pm 0.3^{* *}$ \\
\hline$-\mathrm{PD}$ & 33 & $1,136 \pm 228$ & $988 \pm 94$ & $2.2 \pm 0.4$ & $1.9 \pm 0.4$ \\
\hline $\begin{array}{l}\text { MLT+5- } \\
\text { MTT+PNL }\end{array}$ & 28 & $1,284 \pm 218$ & $1,467 \pm 188$ & $2.1 \pm 0.5$ & $2.7 \pm 0.4$ \\
\hline$-\mathrm{DC}$ & 22 & $1,637 \pm 158$ & $1,843 \pm 145$ & $2.3 \pm 0.5$ & $3.5+1-0.3^{* * *}$ \\
\hline$-\mathrm{PD}$ & 6 & $1,093 \pm 287$ & $941 \pm 134$ & $1.9 \pm 0.4$ & $1.6 \pm 0.5$ \\
\hline
\end{tabular}

${ }^{*} \mathrm{P}<0.05$ vs patients with $\mathrm{PD} ;{ }^{* *} \mathrm{P}<0.01$ vs patients with $\mathrm{PD}$.

related pineal deficiency, which could involve the whole endocrine activity of pineal itself [21]. This finding is not surprising, since cancer progression would not be characterized by a decline in MLT nocturnal production, but also by a progressive pineal histological damage, with a following probabl deficiency of antitumor pineal hormones other than MLT, namely 5-MTT and PNL. In fact, in tumor-bearing pinealectomized animals, the exogenous administration of MLT has appeard to abolish some but not the overall pro-tumoral effects induced by the pinealectomy, by confirming the existence of pineal antitumor hormones other than MLT itself [26]. If successive randomized studies will confirm the greater anticancer efficacy of the administration of more pineal antitumor hormones with respect to the only MLT, the total pineal endocrine replacement therapy could consitute a new simple inexpensive non-toxic cancer cure to achieve a control of the neoplastic growth by restablishing the physiological neuroendocrine mechanisms responsible for the neuroendocrine control of cancer onset and growth in the health conditions, mainly played by the pineal, though either an inhibitory effects on cancer cells proliferation, or by piloting the immmune responses in an antitumor way. In fact, in addition to the well demonstrated inhibitory effets of several pineal hormones on cancer cell proliferation and dissemination, this preliminary study would suggest that the pineal hormones may act in an antitumor way by also improving the immune status of patients, because of the evidence that the control of disease was constantly associated with an improvement in the antitumor immunity, as shown by the increase in patients, who achieved disease stabilization or tumor regression in both lymphocyte count and LMR values, which are two common prognostic biomarkers to evaluate the immune status of cancer patients and to predict their prognosis [25].

\section{References}

1. Antony MH (1998) Psychoneuroimmunology of cancer. Brain Behav Immunol 17: 84 91.

2. Mantovani A, Allavena P, Sica A, Balkwill F (2008) Cancer-related inflammation. Nature 454: 436-444. [Crossref]

3. Zou W (2006) Regulatory T cells, tumour immunity and immunotherapy. Nat Rev Immunol 6: 295-307. [Crossref]

4. Ashwell S, Janetka JW, Zabludoff S (2008) Keeping checkpoint kinases in line: new selective inhibitors in clinical trials. Expert Opin Investig Drugs 17: 1331-1340. [Crossref]

5. Chen J, Jiang CC, Jin L, Zhang XD (2016) Regulation of PD-L1: a novel role of prosurvival signalling in cancer. Ann Oncol 27: 409-416. [Crossref]

6. Dejaco C, Duftner C, Schirmer M (2006) Are regulatory T-cells linked with aging? Exp Gerontol 41: 339-345. [Crossref]

7. Grimm EA, Mazumder A, Zhang HZ, Rosenberg SA (1982) Lymphokine-activated killer cell phenomenon. Lysis of natural killer-resistant fresh solid tumor cells by interleukin 2-activated autologous human peripheral blood lymphocytes. $J$ Exp Med 155: 1823-1841. [Crossref]

8. Lissoni P, Mandalà M, Rovelli F, Giani L, Barni S, et al. (1998) Effect of interleukin-12 immunotherapy on endogenous secretion of interleukin-2 in metastatic cancer patients. Int J immunother XIV: 101-104,

9. Maestroni GJ (1993) The immunoneuroendocrine role of melatonin. J Pineal Res 14 1-10. [Crossref]

10. Brzezinski A (1997) Melatonin in humans. N Engl J Med 336: 186-195. [Crossref]

11. Lissoni P (1999) The pineal gland as a central regulator of cytokine network. Neuro Endocrinol Lett 20: 343-349. [Crossref]

12. Lewis JW, Shavit Y, Terman GW, Nelson IR, Gale RP, et al. (1983) Apparent involvement of opioid peptides in stress-induced enhancement of tumor growth Peptides 4: 635-638.

13. Regelson W, Pierpaoli W (1987) Melatonin: a rediscovered antitumor hormone? Its relation to surface receptors; sex steroid metabolism, immunologic response, and chronobiologic factors in tumor growth and therapy. Cancer Invest 5: 379-385. [Crossref] 
Lissoni P (2019) A study of a possible cancer cure by correcting the main cancer-related psychoneuroendocrine alterations responsible for tumor-related immunosuppression

14. Sze SF, Ng TB, Liu WK (1993) Antiproliferative effect of pineal indoles on cultured tumor cell lines. J Pineal Res 14: 27-33. [Crossref]

15. Pless G, Frederiksen TJ, Garcia JJ, Reiter R (1999) Pharmacological aspects of $\mathrm{N}$-acetyl-5-methoxytryptamine (melatonin) and 6-methoxy-1,2,3,4-tetrahydro-betacarboline (pinoline) as antioxidants: Reduction of oxidative damage in brain region homogenates. J Pineal Res 26: 236-246.

16. Bartsch C, Bartsch H (1999) Melatonin in cancer patients and in tumor-bearing animals. Adv Exp Med Biol 467: 247-264. [Crossref]

17. Fumagalli L, Lissoni P, Di Felice G, Meregalli S, Valsuani G, et al. (1999) Pretreatment serum markers and lymphocyte response to interleukin-2 therapy. Br J Cancer 80: 407411. [Crossref]

18. Lissoni P (2002) Is there a role for melatonin in supportive care? Support Care Cancer 10: 110-116. [Crossref]

19. Lissoni P (2014) Melatonin in human cancer: therapeutic possibilities. In: Melatonin and melatoninergic drugs and clinical practice. Springer India, pp: 43-56.
20. Conti A, Maestroni GJ (1995) The clinical neuroimmunotherapeutic role of melatonin in oncology. J Pineal Res 19: 103-110. [Crossref]

21. Hajdu SI, Porro RS, Lieberman PH, Foote FW Jr (1972) Degeneration of the pineal gland of patients with cancer. Cancer 29: 706-709. [Crossref]

22. Winters WD, Benavides R, Clause VJ (1981) Effects of Aloe extracts on normaslans tumour cells in vitro. Econ Botany 35: 89-95.

23. Shishodia S, Harikumar KB, Das S, Ramawat KG, Aggarwal BB (2008) The guggu for chronic diseases: ancient medicine, modern targts. Anticancer Res 28: 3647-3664.

24. Fried LE, Arbiser JL (2009) Honokiol, a multifunctional antiangiogenic and antitumor agent. Antioxid Redox Signal 11: 1139-1148. [Crossref]

25. Eo WK, Chang HJ, Kwon SH, Koh SB, Kim YO, et al. (2016) The lymphocyte-tomonocyte ratio predicts patient survival and aggressiveness of ovarian cancer. $J$ Cancer 7: $289-296$

26. el-Domeiri AA, Das Gupta TK (1973) Reversal by melatonin of the effect of pinealectomy on tumor growth. Cancer Res 33: 2830-2833. [Crossref]

Copyright: (2019 Lissoni P. This is an open-access article distributed under the terms of the Creative Commons Attribution License, which permits unrestricted use, distribution, and reproduction in any medium, provided the original author and source are credited. 